\title{
Body-image and -size perception after a single session of HIIT body work in healthy adult men
}

\author{
Roberta Luksevicius Rica1,2*, João Marcelo de Queiroz Miranda1, Alexandre Fernandes \\ Machado $^{1}$, Alexandre Lopes Evangelista ${ }^{3}$, Cauê Vazquez La Scala Teixeira4 ${ }^{4}$, Eliane Florencio \\ Gama $^{1}$, Graça Pinto ${ }^{5}$, Victor Machado Reis ${ }^{5}$, Danilo Sales Bocalini ${ }^{6}$
}

It was our objective to analyze the effects of a single session of whole body HIIT on body-image and -scheme parameters of healthy adult men. Twenty-one active, healthy adults performed high-intensity intermittent training based on full body exercise. The training session involved 20 sets of 30 second all-out exercise and 30 seconds of passive recovery between sets. All subjects underwent blood lactate measurement, rate of perceived exertion, feeling scale and body image assessment and perception of body size measurements. Significant differences $(p<0.001)$ were found on blood lactate but not in feeling scale. The values of current and adequate silhouette did not differ between each other and the real silhouette. Significant differences were found on Image Marking Procedure (IMP) $(p<0.003)$. Additionally, significant differences $(p=0.009)$ were found on prevalence of adequate, hypoeschematic and hypeschematic. Although alteration was not found on self-perception of body image, a single session of HIIT body work promoted alteration on body size perception in healthy adult men.

Keywords: whole-body, HIIT, body scheme, body image.

\section{INTRODUCTION}

Body perception (BP) can be conceptualized as the integration of sensory, conceptual and idea information that the subject has about their body in a dynamic process that inter-relates the body with the environment (De Vignemont, 2010). To Ivanenko et al. (2011), the perception of the dimensions of the body and its segments is essential to adequate movement in space. In addition, it is also necessary to perceive the position of each segment and the body as a whole, to elaborate a program of action and to recognize the position of the body in relation to space and movable and immovable objects (Ivanenko et al. 2011). Studies (Tsakiris et al., 2005; Tsakiris, Prabhu \& Haggard, 2006) suggest that tactile stimulation, passive movement and active movement are important sources of stimulus for the development of the body perception; however, sensorial information contributes with the construction of representations of individual parts of the body while the active movement contributes with an overall corporal representation. This information suggests that $\mathrm{BP}$, as a whole, depends fundamentally more on the movement and on the sensation (Tsakiris et al., 2005; Tsakiris, Prabhu \& Haggard, 2006). In addition to being one of the important elements in the construction of body representation, participation in regular physical activity is associated with benefits in the variation of health status (Coratella \& Schena, 2016; Distefano et al., 2013).

\footnotetext{
Manuscript received at April 15 ${ }^{\text {th }}$ 2016; Accepted at December $8^{\text {th }} 2016$

${ }^{1}$ Programa de Pós-Graduação em Educação Física, Universidade São Judas Tadeu, São Paulo, SP, Brasil.

${ }^{2}$ Universidade Cidade de São Paulo. Grupo de Estudo em Pedagogia do Esporte e Movimento. São Paulo, SP, Brasil.

${ }^{3}$ Universidade Nove de Julho. Departamento de Educação, Curso de Educação Física, São Paulo, SP, Brasil.

${ }^{4}$ Faculdade Praia Grande. Faculdade de Educação Física, Praia Grande, SP, Brasil.

${ }^{5}$ Universidade de Trás-os-Montes e Alto Douro. Centro de Investigação em Desporto, Saúde e Desenvolvimento Humano, CIDESD, Vila Real, Portugal

${ }^{6}$ Universidade Federal do Espírito Santo. Laboratorio de Fisiologia e Bioquimia Experimental do Centro de Educação Física e Desporto, Vitória, ES, Brasil.

* Corresponding author: Rua Militão Barbosa de Lima, 56, 09720-420, Centro, SBC, SP, Brasil

email: robertarica@hotmail.com
} 
Among the currently available physical activity strategies, high intensity interval training (HIIT) has been considered an efficient approach to reduce cardiometabolic risk (Falcone et al., 2015; Shaban, Kenno \& Milne, 2014) and increase in sports performance (Naimo et al., 2015). Recently, Machado et al. (2017) proposed the use of exercises using whole body in HIIT (HIIT body work) programs. According to Thompson (2017) the training programs using body weight have been identified as a strong trend in recent years, mainly for providing independence for exercise performance, low operating cost and freedom of three-dimensional movement. Previous studies (Bellenger et al., 2016; Gist et al., 2015;) have suggested that HIIT body work offers physiological benefits similar to those observed in traditional HIIT protocols.

Although, physical activity has been considered a key element of health promotion and improvement of overall physical fitness as well as an approach to increase body selfknowledge, self-esteem, self-perception and effectiveness, which are important factors for a positive body image in different populations (Bizerra \& Gama 2017; Fonseca et al., 2014; Pereira \& Gama, 2017; Rica et al., 2018a; Rica et al., 2018b). However, to the best of our knowledge, there is a paucity of information between HIIT and self-perception of body image. Thus, the aim of this study was to evaluate the influence of a single session of HIIT body work in self-perception of body image in adult men.

\section{METHOD}

\section{Participants}

After the approval of the São Judas Tadeu University Research Ethics Committee $\left(\mathrm{n}^{\circ}\right.$ 1.738.246/2016), a consent document was signed by twenty healthy adult men $(28 \pm 5$ years), who were physically independent and volunteered to participate in this study. The following parameters were used as exclusion criteria: positive clinical diagnosis of diabetes mellitus, smoking, musculoskeletal complications and/or cardiovascular alterations confirmed by medical evaluation. All the procedures were in accordance with the ethical standards of the responsible committee on human experimentation (institutional or regional) and with the Helsinki Declaration of 1975, as revised in 1983.

\section{Procedures}

A single acute bout of high-intensity interval training based on full body exercise was performed according previously studies (Machado et al., 2018a; Machado et al., 2018b). Briefly, the training session involved a $5 \mathrm{~min}$ warm-up followed by 20 sets of 30 seconds of allout exercise and 30 seconds of passive recovery between sets, totaling 25 minutes of exercise. The following exercise order ( 5 sets for each exercise) was used for the jumping jack, burpee, mountain climb and squat jump.

Height was measured by a Cardiomed (WCS model) stadiometer, with an accuracy of 115/220 $\mathrm{cm}$. The measurement was performed with the cursor at an angle of $90^{\circ}$ with the patient in a standing position with feet together in contact with the Stadiometer. The subjects were instructed to stay in inspiratory apnea, with the head parallel to the ground. Total body mass was measured by a calibrated Filizola electronic scale (Personal Line Model 150) with a 100g scale and a maximum capacity of $150 \mathrm{~kg}$. Body mass index (BMI, $\mathrm{kg} / \mathrm{m}^{2}$ ) was calculated using the equation BMI $=$ weight $/$ height $^{2}$. The general sample characteristic parameters assessed are described in Table 1.

Table 1. Anthropometric characteristics.

\begin{tabular}{lcc}
\hline \multicolumn{1}{c}{ Parameters } & Mean \pm SD & $95 \%$ CI \\
\hline Body mass (kg) & $78.41 \pm 10.51$ & $73.63-83.20$ \\
Height $(\mathrm{m})$ & $1.76 \pm 0.08$ & $1.72-1.79$ \\
BMI $(\mathrm{kg} / \mathrm{m} 2)$ & $25.24 \pm 2.64$ & $24.03-26.44$ \\
\hline
\end{tabular}

$\overline{\text { Values expressed in mean } \pm \text { standard deviation. BMI: body }}$ mass index.

The total exercise movement amount realized for each set was used as external training load, as suggested by Machado et al. (2017a) and utilized in other studies (Machado et al. 2018a, Machado et al., 2018b). The number of movements realized for each set were counted and summed at final exercise session.

Capillary blood samples were taken from a sterile fingertip using a sterile lancet before and after the exercise session according to previous 
publication of our group (Machado et al., 2018a; Machado et al., 2018b). The first drop of blood was discarded, and freeflow blood was collected in glass capillary tubes. All blood samples $(25 \mathrm{ml})$ for lactate analysis were evaluated using an Accutrend ${ }^{\circledR}$ (Roche - Basel, Switzerland) lactate analyzer.

Subjects reported their rating of perceived exertion (RPE) using a Borg (0-10 scale) after a single session of HIIT body work according previously studies (Machado et al., 2018a; Machado et al., 2018b). To guarantee that the RPE mean data obtained refers to the total training, subjects were asked to answer a question 20 to 30 minutes after the end of the session: "How was your training today"?

The body-image and -satisfaction was analyzed utilizing previously study (Kakeshita et al, 2009). This task consists of 12 silhouettes on a progressive scale, been the discrepancy between the current (CS) and ideal silhouettes (IS) was analyzed after the figures were presented to the subjects. According to study of four group (Rica et al. 2018a) the following questions were directed to the men: (1) What is the silhouette that best represents your current physical appearance? (2) What silhouette would you like to have?

Body schema was measured according to previously publications (Fonseca et al., 2012). Briefly, using the Image Marking Procedure (IMP), evaluation was made through the sense of touch at certain spots. Before and after whole body HIIT single session, all participants were marked with masking tape on the right and left acromioclavicular joint, right and left waist curves, and greater trochanter of the right and left femur with green, blue, red, and black round stickers to ensure that the same points were always touched by the researcher in all three trials. Participants remained in standing position near a wall. The wall had a measuring tape placed vertically for the researcher's use as reference. The test was performed blindfolded. The researcher touched the points marked on the participant's body and the participants had to point the orthogonal projection of the touched point on the wall. The researcher marked each indicated point with a colored label. The first point touched was the top of the head, followed by the right shoulder, waist, and hip, and then the left shoulder, waist, and hip. The test was repeated three times, and the participant could not see the previous markings. After collecting the perceived measurements, the examiner guided the individual close to the wall, so that the anterior foot alignment was maintained. In this position, the marking of veridical heights of body points was performed using the measuring tape. The labels and measuring tape on the wall were photographed with a digital camera for further analysis using AxioVision Version 3.1.

The quantitative analyses of IMP were evaluated by distances of the points identified by the subject and the evaluator for body height and width in the horizontal plane. Data were organized in tables and the overall body perception index (BPI) was calculated. First, the BPI of each region was analyzed. From the three attempts of perceived measures for each region, perceived size was calculated as the mean response divided by actual measurement, multiplied by 100. Overall BPI (oBPI) was calculated as the mean BPI of measurement sites [ (head BPI + shoulder BPI + waist BPI + hip BPI) / 4]. Perception was defined as when the actual and perceived measures were very similar, with BPI between $99.4 \%$ and $112.3 \%$. Overall body awareness was defined as adequate, i.e., when the perceived measures are very close to the actual measurements, with BPI between $99.4 \%$ and $112.3 \%$. When the perceived measure differed from the actual measurement, individuals were classified as hyperschematic (oBPI greater than $112.3 \%$ ) or as hyposchematic (oBPI less than 99.4\%).

\section{Statistical analysis}

The D'Agostino-Pearson test was applied to Gaussian distribution analysis. The paired Student's t-test was performed to compare differences before and after whole body HIIT single session. An alpha of 0.05 was used to determine statistical significance. All data values were expressed as a mean \pm standard deviation. All analyses were performed using SPSS software (v 15.0; IBM, Armonk, NY, USA). 


\section{RESULTS}

The participants presented no injuries as a result of the workout during and after the exercise session. As foreseen, due to high exercise intensity, the values of the rate of perceived exertion $(9 \pm 1)$ and total amount of movements (503 \pm 123 numbers) were highest. Additionally, the blood lactate concentration increased $(953 \pm 338 \%)$ after whole body HIIT single session. As shown in Figure 1, significant differences $(p<0.001)$ were found between (Before: $1.22 \pm 0.25$, After: 12.45 C 3.47; $\mathrm{mMol} / \mathrm{L}$ ) of blood lactate.

The values of actual (Before: $5.95 \pm 2.22$, After: $5.71 \pm 2.53, p=0.6339$ ) and ideal (Before: $6.04 \pm 1.71$, After: $5.33 \pm 1.90, p=0.0744) \mathrm{did}$ not differ each other (Figure 2A) and real silhouette $(6.09 \pm 1.04)$. Similar results were found $(p=0.3114)$ on the body satisfaction (Before: $-0.09 \pm 1.99$, After: $0.47 \pm 3.31$ ) and prevalence of body satisfaction as showed at Figure 2B.

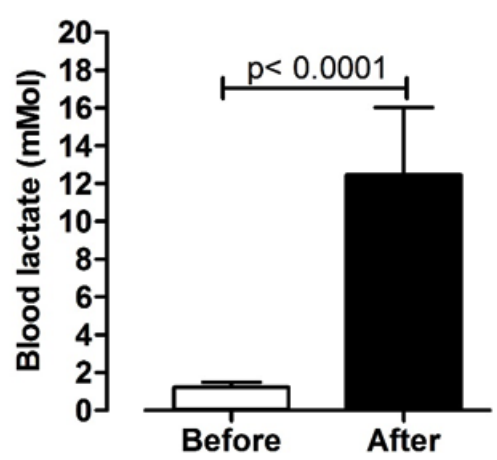

Figure 1. Values are presented as a mean \pm standard deviation of blood lactate concentration before and after HIIT body work single session.
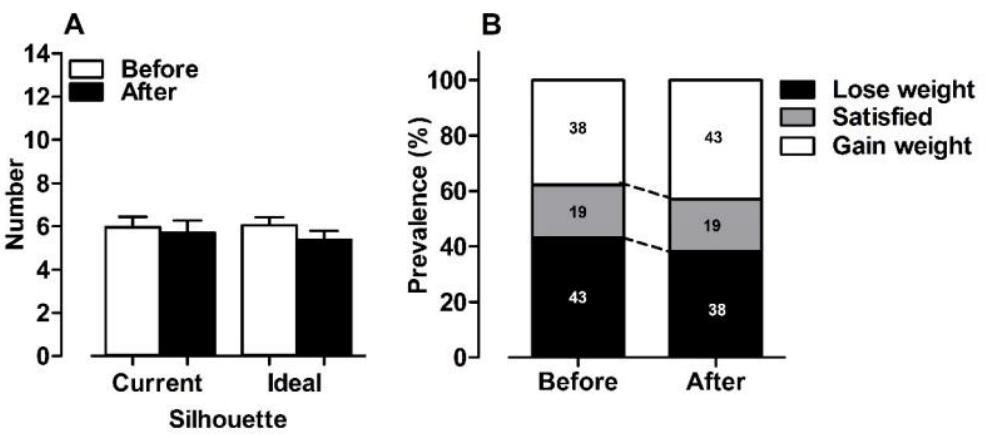

Figure 2. Values are presented as a mean \pm standard deviation before and after HIIT body work single session of current and adequate silhouette (Panel A) and prevalence of body satisfaction (Panel B).

The values of IMP increase $(13.46 \pm 17.72 \%)$ after whole body HIIT single session. As showed in Figure 3A significant differences was found (Before: $96.18 \pm 11.57$, After: $109.65 \pm 12.13$, $\mathrm{p}<0.003)$, moreover, significant differences $(p=$
0.009) were found on prevalence of body size classification as showed in Figure 3B. Additionally, a significant correlation $(\mathrm{p}=$ 0.0073, $r=0.4079$ ) was found on IMP and blood lactate concentration (Figure $3 \mathrm{C}$ ).
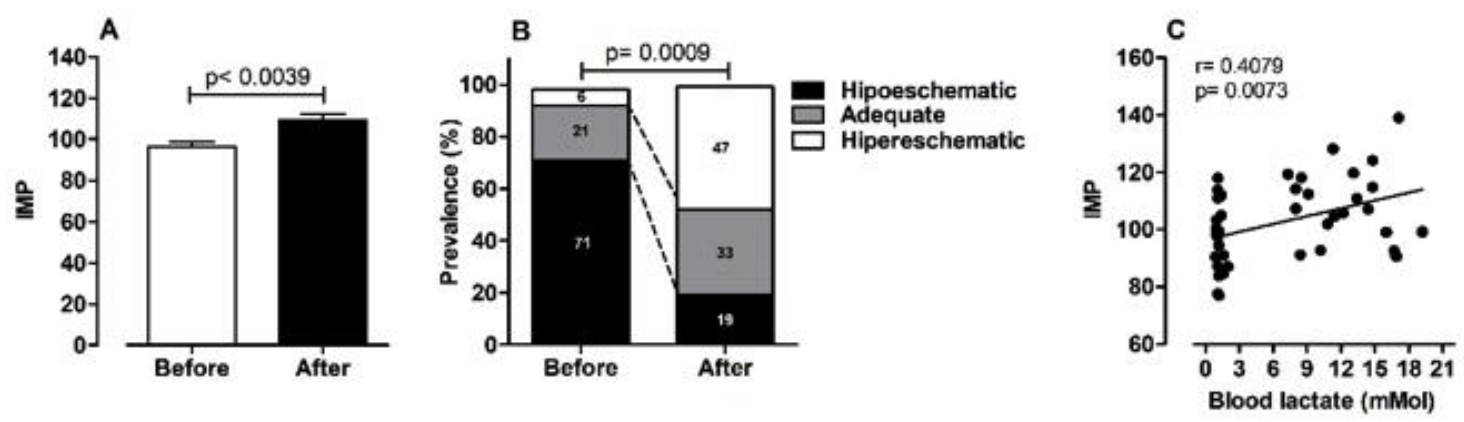

Figure 3. Values are presented as a mean \pm standard deviation before and after HIIT body work single session of IMP (Panel A), prevalence of body size classification (Panel B) and correlation of IMP and blood lactate concentration (Panel C). 


\section{DISCUSSION}

With the increase in popularity of HIIT and exercise with body weight, there is a growing need for studies to analyze the effects of interventions that combine the two strategies on the psychobiological variables (Evangelista et al., 2017; Machado et al., 2018a; Machado et al., 2018b; Machado et al., 2018c,). In this study, the main results were no self-perception of body image change, statistical difference on perception of body size and a positive correlation between IMP and blood lactate after HIT bodywork. To the best of our knowledge, this is the first report to describe self-perception of body-image and size after HIIT approach in adult men.

In our study the blood lactate concentration, total amount of movement and rate of perceived exertion increased significantly, which has been shown in previous studies (Machado el al., 2018a; Machado et al., 2018c; Evangelista et al., 2017) confirming that this exercise protocol is able to promote fatigue and metabolic acidosis such as other HIIT protocols (Wahl et al., 2010; Nalbandian, Radak \& Takeda, 2017).

The greatest determinants for negative or positive body image are satisfaction with weight, accuracy of size perception, body satisfaction, appearance evaluation and orientation, body esteem, ideal body, body schema and body awareness (Rica et al., 2018a). Further factors also include sex, age, mean and the relationship of the body with cognitive processes such as cultural and social beliefs, values and attitudes (Rica et al., 2018a; Gardner, 2011). In relationship to body image and exercise, studies have shown improvement on body image after acute (Béres, Czeglédi \& Babusa, 2017; Carraro, Nart \& Scarpa, 2010) and chronic (Baştuğ et al., 2016; Vocks et al., 2009) protocols. However, no significant differences were found in our study after a single session of HIIT body work. Similar results have been shown in a meta-analysis study by Alleva et al. (2015), with interventions inducing only small improvements in body image and underline the need for large-scale, highquality trials in this area.

In addition, other important points of view should be addressed for a male sample, exercise modality and session. The silhoute choice by male subjects has been shown to be higher than that of women (Damasceno et al., 2005; Haines \& Neumark-Sztainer, 2006). However, in our study no diffeterences were found on current, ideal and real silhouettes, different than others. Additionally, to the best of our knowledge, this is the first study that evaluates body image after whole body HIIT session.

There are few studies (Pereira 2017) that have investigated the influence of high intensity exercises and BP. In our study, the HIIT body work session changed $13.46 \%$ on perception of body size, different than that of Pereira (2017), evaluating Cross Fit practitioners after a 9minute session of high intensity exercises, who evidenced an increase of $3 \%$ in the perception of the body. However, unlike the study by Pereira (2017), we showed a significant difference in the classification of body perception. There are reports in the literature that body activities are capable of modifying body dimensional perception (Catalan-Matamoros et al., 2011; Hedlund \& Gyllensten, 2010), but using highintensity exercises, the possible mechanisms still remain scarce.

To Pereira (2017), training can provide neural stimuli necessary to promote the modification of the perception of the dimension after training, mainly by the increase of muscular tone and in regions in which it does not have bony limits or when the musculature undergoes constant stimuli. The findings of previously studies (Tsakiris et al., 2005; Tsakiris, Prabhu \& Haggard, 2006) may help us in understanding the data obtained in this study. These studies (Tsakiris et al., 2005; Tsakiris, Prabhu \& Haggard, 2006) suggest different mechanisms of body information processing in the primary somesthetic cortex and the primary motor cortex. While the somesthetic cortex corresponds to the representation of a well delimited cutaneous segment, the representations of different parts of the body overlap in the motor cortex. According to Lemon (1998) the primary motor cortex seems to organize itself to represent muscle groups and synergies of movement rather than individual muscles. It is speculated that given the multi- 
articular and synergistic characteristic of HIIT, a large motor cortical area is stimulated, which may explain the overestimation of body size (hyperskema).

Another possible explanation for the changes in the perception of the dimension can be directed to the acute modifications derived from the practice of exercise, thus, it is possible to hypothesize that the exercises selected in the present study, because they are performed with the whole body and with all-out intensity, can induce systemic changes, especially when associated with metabolic demand. Among the acute mechanisms we can highlight is the hyperemia induced by the exercise. Hyperemia can conceptually be considered as an increase in blood flow in a given region, provided by vasodilation mainly for maintenance of function, nutrition and tissue oxygenation (Joyner \& Casey, 2015; Wunsch, Muller-Delp \& Delp, 2000), in addition, the magnitude of hyperemia is associated with intensity of the exercise, and thus, of the request of activity and muscular action and metabolic alterations as showed on figure $2 \mathrm{C}$.

\section{CONCLUSION}

The results of present study suggest that although the single session of HIIT body work exercise does not promote self-perception of body image change, it induces alterations on perception of body size in healthy adult men with association of blood lactate concentration.

\section{Acknowledgments:}

Nothing to declare.

Conflict of interests:

Nothing to declare.

\section{Funding:}

Nothing to declare.

\section{REFERENCES}

Alleva, J. M., Sheeran, P., Webb, T. L., Martijn, C., \& Miles, E. (2015). A meta-analytic review of standalone interventions to improve body image. PLoS One, 10(9), e0139177.
Baştuğ, G., Özcan, R., Gültekin, D., \& Günay, Ö. (2016). The effects of cross-fit, pilates and zumba exercise on body composition and body image of women. International Journal of Sports, Exercise and Training Science, 2(1), 22-29.

Bellenger, C. R., Thomson, R. L., Howe, P. R., Karavirta, L., \& Buckley, J. D. (2016). Monitoring athletic training status using the maximal rate of heart rate increase. Journal of science and medicine in sport, 19(7), 590-595.

Béres, A., Czeglédi, E., \& Babusa, B. (2017). Effects of a single aerobic exercise session on body image. Mentálhigiéné és Pszichoszomatika, 18(1), 84-104.

Bizerra, A., \& Gama, E. F. (2017). Neurocognitive aspects of body size estimation-A study of contemporary dancers. Motriz: Revista de Educação Física, 23(1), 33-39.

Catalan-Matamoros, D., Helvik-Skjaerven, L., LabajosManzanares, M. T., Martínez-de-SalazarArboleas, A., \& Sánchez-Guerrero, E. (2011). A pilot study on the effect of Basic Body Awareness Therapy in patients with eating disorders: a randomized controlled trial. Clinical Rehabilitation, 25(7), 617-626.

Carraro, A., Nart, A., \& Scarpa, S. (2010). Effects of a single session of physical exercise on body state image. Revista Brasileira de Ciências do Esporte, 32(1), 173-184.

Coratella, G., \& Schena, F. (2016). Eccentric resistance training increases and retains maximal strength, muscle endurance, and hypertrophy in trained men. Applied Physiology, Nutrition, and Metabolism, 41(11), 1184-1189.

Damasceno, V. O., Lima, J. R. P., Vianna, J. M., Vianna, V. R. Á., \& Novaes, J. S. (2005). Tipo físico ideal e satisfação com a imagem corporal de praticantes de caminhada. Revista braileira de medicina do esporte, 11(3), 181-6.

De Vignemont, F. (2010). Body schema and body image-Pros and cons. Neuropsychologia, 48(3), 669-680.

DiStefano, L. J., DiStefano, M. J., Frank, B. S., Clark, M. A., \& Padua, D. A. (2013). Comparison of integrated and isolated training on performance measures and neuromuscular control. The Journal of Strength \& Conditioning Research, 27(4), 10831090.

Evangelista, A. L., de Toledo, R. A. G., Evangelista, R. L. R., Fernandes, A., Machado, J. M. Q., \& La Scala, C. V. (2017). Effects of High-Intensity Calisthenic Training on Mood and Affective Responses. Journal of Exercise Physiology Online, 20(6), 15-24.

Falcone, P. H., Tai, C. Y., Carson, L. R., Joy, J. M., Mosman, M. M., McCann, T. R., ... \& Moon, J. R. (2015). Caloric expenditure of aerobic, resistance, or combined high-intensity interval training using a hydraulic resistance system in healthy men. The Journal of Strength $\mathcal{E}$ Conditioning Research, 29(3), 779-785.

Fonseca, C. C., Gama, E. F., Thurm, B. E., Pereira, E. S., Limongelli, A. M. D. A., \& Miranda, M. L. D. 
J. (2012). Benefits of body perception stimulation on body scheme among the elderly. Revista Brasileira de Geriatria e Gerontologia, 15(2), 353364.

Fonseca, C. C., Thurm, B. E., Vecchi, R. L., \& Gama, E. F. (2014). Ballroom dance and body size perception. Perceptual and motor skills, 119(2), 495503.

Gardner, R. M. (2011). what Affects Body Size Estimation? The Role of Eating Disorders, Obesity, Weight Loss, Hunger, Restrained Eating, Mood, Depression, Sexual Abuse, Menstrual Cycle, Media Influences, and Gender. Current Psychiatry Reviews, 7(2), 96-103.

Gist, N. H., Freese, E. C., Ryan, T. E., \& Cureton, K. J. (2015). Effects of low-volume, high-intensity whole-body calisthenics on army ROTC cadets. Military medicine, 180(5), 492-498.

Haines, J., \& Neumark-Sztainer, D. (2006). Prevention of obesity and eating disorders: a consideration of shared risk factors. Health education research, 21 (6), 770-782.

Hedlund, L., \& Gyllensten, A. L. (2010). The experiences of basic body awareness therapy in patients with schizophrenia. Journal of bodywork and movement therapies, 14(3), 245-254.

Ivanenko, Y. P., Dominici, N., Daprati, E., Nico, D., Cappellini, G., \& Lacquaniti, F. (2011). Locomotor body scheme. Human movement science, 30(2), 341-351.

Joyner, M. J., \& Casey, D. P. (2015). Regulation of increased blood flow (hyperemia) to muscles during exercise: a hierarchy of competing physiological needs. Physiological reviews, 95(2), 549-601.

Kakeshita, I. S., Silva, A. I. P., Zanatta, D. P., \& Almeida, S. S. (2009). Construção e fidedignidade teste-reteste de escalas de silhuetas brasileiras para adultos e crianças. Psicologia: Teoria e Pesquisa, 25(2), 263-270.

Lemon, R. (1988). The output map of the primate motor cortex. Trends in Neuroscience, $11(11), 501-$ 506.

Machado, A. F., Baker, J. S., Figueira-Júnior, A. J., \& Bocalini, D. S. (2017). High-intensity interval training using whole-body exercises: training recommendations and methodological overview. Clinical Physiology and Functional Imaging. Ahead of print.

Machado, A. F., Evangelista, A. L., Miranda, J. M. D. Q., Teixeira, C. V. L. S., Leite, G. D. S., Rica, R. L., \& Bocalini, D. S. (2018a). Sweat rate measurements after high intensity interval training using body weight. Revista Brasileira de Medicina do Esporte, 24(3), 197-201.

Machado, A. F., Miranda, M. L. D. J., Rica, R. L., Figueira Junior, A., \& Bocalini, D. S. (2018b). Bodyweight high-intensity interval training: a systematic review. Revista Brasileira de Medicina do Esporte, 24(3), 234-237.

Machado, A. F., Evangelista, A., Miranda, J. M., Teixeira, C., Rica, R. L., Lopes, C. R., Junior, A.
F., Baker, J. S., Bocalini, D. S. (2018c). Description of training loads using whole body exercise during high-intensity-interval-training interval training. Clinics, Ahead of print.

Naimo, M. A., de Souza, E. O., Wilson, J. M., Carpenter, A. L., Gilchrist, P., Lowery, R. P., Averbuch, B., White, T. M., \& Joy, J. (2015). High-intensity interval training has positive effects on performance in ice hockey players. International Journal of Sports Medicine, 36(1), 6166.

Nalbandian, H. M., Radak, Z., \& Takeda, M. (2017). Active Recovery between Interval Bouts Reduces Blood Lactate While Improving Subsequent Exercise Performance in Trained Men. Sports, 5(2), pii: E40.

Pereira, E. S., \& Gama, E. F. (2017). Imagem corporal da mulher praticante de exercício físico. $H U$ Revista, 43(1), 5-11.

Pereira, E. S. (2017). Acute effect of a CrossFit training session on serum BDNF levels, mood states and body perception in active individuals Thesis (doctorate degree) - São Judas Tadeu University, São Paulo.

Rica, R. L., Gama E. F., Machado, A. F., Angelica Castilho Alonso, A. C., Evangelista, A. L., Figueira-Junior, A. I, Zanetti, M., Brandão, R. M., Miranda, M. L. J., Alves, J. V., Bergamin, M., Bocalini, D. S. (2018a) Does resistance training improve body image satisfaction among the elderly? A cross-sectional study. Clinics, ahead of print

Rica, R. L., Bocalini, D. S., de Jesus Miranda, M. L., Valenti, V. E., \& Gama, E. F. (2018b) Imagem corporal de adolescentes do sexo feminino saudáveis e sua associação com a atividade física: revisão sistemática. Body image of healthy adolescent women and its association with physical activity: a systematic review. Ciencia $e$ Saude Coletiva, Ahead of print.

Souza, A. L. L., Gama, E.F., Pereira, E. S., Santiago, F. G., Bandeira, L. J. S. (2015). Relação entre a imagem corporal e o índice de massa corporal de mulheres obesas candidatas à cirurgia de redução do estômago. Motricidade, 11 (S1): 20-25.

Shaban, N., Kenno, K. A., \& Milne, K. J. (2014). The effects of a 2 week modified high intensity interval training program on the homeostatic model of insulin resistance (HOMA-IR) in adults with type 2 diabetes. The Journal of sports medicine and physical fitness, 54(2), 203-209.

Thompson, W. R. (2017). Worldwide survey of fitness trends for 2018: the CREP edition. ACSM's Health \& Fitness Journal, 21 (6), 10-19.

Tsakiris, M., Haggard, P., Franck, N., Mainy, N., \& Sirigu, A. (2005). A specific role for efferent information in self-recognition. Cognition, 96(3), 215-231.

Tsakiris, M., Prabhu, G., \& Haggard, P. (2006). Having a body versus moving your body: How agency structures body-ownership. Consciousness and cognition, 15(2), 423-432. 
Vocks, S., Hechler, T., Rohrig, S., \& Legenbauer, T. (2009). Effects of a physical exercise session on state body image: The influence of preexperimental body dissatisfaction and concerns about weight and shape. Psychology and Health, 24(6), 713-728.

Wahl, P., Zinner, C., Achtzehn, S., Bloch, W., \& Mester, J. (2010). Effect of high-and low-intensity exercise and metabolic acidosis on levels of $\mathrm{GH}$, IGF-I, IGFBP-3 and cortisol. Growth Hormone \& IGF Research, 20(5), 380-385.

Wunsch, S. A., Muller-Delp, J., \& Delp, M. D. (2000). Time course of vasodilatory responses in skeletal muscle arterioles: role in hyperemia at onset of exercise. American Journal of Physiology-Heart and Circulatory Physiology, 279(4), H1715-H1723.

All content of Journal Motricidade is licensed under Creative Commons, except when otherwise specified and in content retrieved from other bibliographic sources. 